\section{Triterpenos de Hyptis fasciculata Benth.}

\section{Falcão, D.Q.; Fernandes, S.B.O.; Menezes, F.S.*}

Departamento de Produtos Naturais e Alimentos, Faculdade de Farmácia, Centro de Ciências da Saúde, Universidade Federal do Rio de Janeiro

\section{Resumo}

Partes aéreas do arbusto Hyptis fasciculata Benth. foram estudadas do ponto de vista químico. Após vários processos cromatográficos em coluna de gel de sílica, foi possível isolar um triterpeno livre (ácido betulínico), além de triterpenos em mistura (ácido ursólico com ácido betulínico e ácido ursólico com ácido oleanólico), esteróides em mistura (sitosterol e estigmasterol) e misturas de estigmasterol com ácido caféico e sitosterol com lignana. Tais substâncias foram identificadas por ressonância magnética nuclear de ${ }^{1} \mathrm{He}{ }^{13} \mathrm{C}$.

\begin{abstract}
The species Hyptis fasciculata Benth. is a shrub that is still unknown by the chemical point of view. This species has been studied in our group using their aerial parts. After many cromatographic processes over silica gel column, it was possible to isolate free triterpene (betulinic acid) and triterpenes in mixture (ursolic acid with betulinic acid and ursolic acid with oleanolic acid), one steroid mixture (sitosterol and stigmasterol) and a mixture of steroid with cafeic acid (stigmasterol and cafeic acid) and with lignan (sitosterol and sesamin). These compounds were identified by nuclear magnetic ressonance ${ }^{1} \mathrm{H}$ and ${ }^{13} \mathrm{C}$.
\end{abstract}

O gênero Hyptis é formado por aproximadamente 400 espécies distribuídas desde o sul dos Estados Unidos até a Argentina. Esse gênero pertence à família Lamiaceae, uma vasta família com cerca de 200 gêneros e 3200 espécies, de distribuição cosmopolita, mas centrada, principalmente, na região mediterrânea, onde constitui parte integrante da vegetação ${ }^{1}$. Hyptis é um gênero rico em espécies de grande importância econômica e etnofarmacológica, cujos conhecimentos são transmitidos de geração a geração até os dias de hoje. As populações as utilizam para fins medicinais, não só no Brasil ${ }^{4}$, mas também no México ${ }^{3,5,6,7,8,9}$, Índia ${ }^{10,11} \mathrm{China}^{12}$, Equador ${ }^{13}$, Tailândia ${ }^{13}$, Caribe $^{14}$, Norte da Nigéria ${ }^{15}$ e em outras localidades da África ${ }^{14,16,17}$.

Espécies desse gênero se caracterizam pela presença de substâncias com potencial farmacológico bastante interessante, apresentando uma composição muito rica em óleos essenciais ${ }^{15,16,18}$.
A espécie Hyptis fasciculata Benth. foi coletada nas cercanias de Porto Alegre, no município de Campo Bom, no Rio Grande do Sul, em 1998. Essa espécie, ainda inédita do ponto de vista químico, tem sido estudada em nosso grupo. Foram encontrados um triterpeno livre (ácido betulínico) e triterpenos em mistura (ácido ursólico com ácido betulínico e ácido ursólico com ácido oleanólico), esteróides em mistura (sitosterol com estigmasterol) e misturas de estigmasterol com ácido caféico e sitosterol com sesamina.

Após vários processos cromatográficos foi possível isolar e identificar um triterpeno livre, o ácido betulínico (I), e misturas de triterpenos, ácido ursólico (II) com ácido betulínico e ácido ursólico com ácido oleanólico (III), dentre outras substâncias.<smiles>C=C(C)C1CCC2(C(=O)O)CC[C@]3(C)C(CCC4[C@@]5(C)CCC(O)C(C)(C)C5CC[C@]43C)C12</smiles>

(<smiles>CC1CCCC2(C(=O)O)CC[C@]3(C)C(=CCC4[C@@]5(C)CCC(O)C(C)(C)C5CC[C@]43C)C12</smiles>

( II )<smiles>CC1(C)CCC2(C(=O)O)CC[C@]3(C)C(=CCC4[C@@]5(C)CCC(O)C(C)(C)C5CC[C@]43C)C2C1</smiles>

( III ) 
Fluxograma 1. Isolamento das substâncias presentes no extrato hexânico dos caules de Hyptis fasciculata Benth.

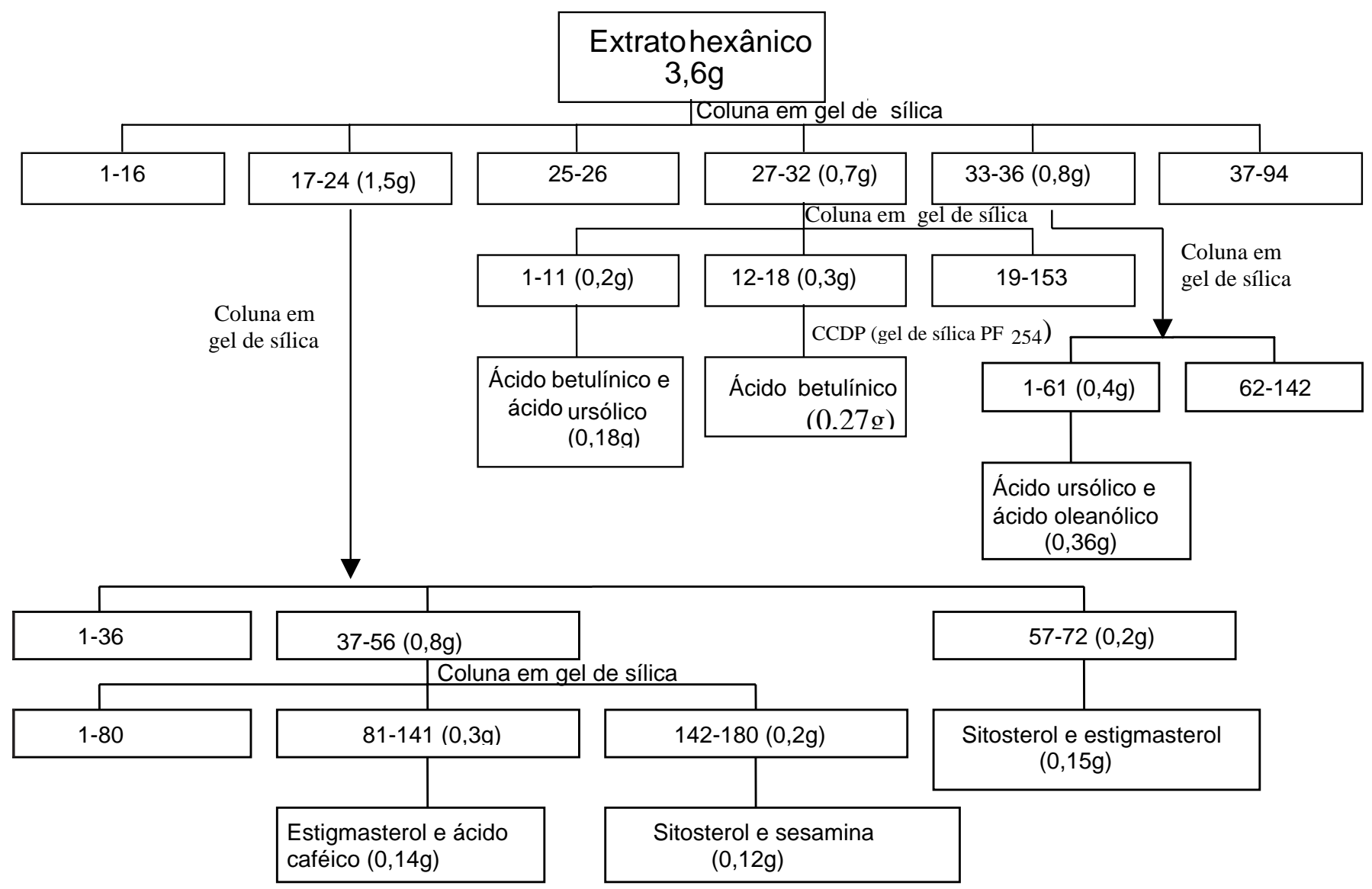

Até o presente momento, com os resultados já obtidos através do estudo fitoquímico do extrato hexânico da Hyptis fasciculata, podemos concluir que as substâncias isoladas e identificadas seguem o padrão encontrado para plantas dessa família, notadamente para o gênero Hyptis onde a ocorrência de triterpenos é elevada.

\section{Material e Métodos}

O material vegetal foi coletado com a ajuda do Dr. Sérgio Bordignon, em Porto Alegre. Para a realização deste estudo fitoquímico foram utilizadas separadamente as partes aéreas (caules e folhas) de Hyptis fasciculata Benth.

A planta foi seca a temperatura ambiente e posteriormente triturada em moinho de facas. Os caules moídos (36g) foram submetidos a processos de extração por maceração estática com etanol $\left(96,5^{\circ} \mathrm{GL}\right)$ por $48 \mathrm{~h}$ (10 vezes). O extrato etanólico obtido foi evaporado separadamente e, em seguida, suspendido em água destilada. Após, foi realizado um processo de extração líquido-líquido com hexano (3,6 g), diclorometano $(2,6 \mathrm{~g})$, acetato de etila $(1,9 \mathrm{~g})$ e n-butanol $(3,0 \mathrm{~g})$.

O extrato hexânico dos caules, foi cromatografado em coluna de gel de sílica utilizando-se gradientes crescentes de hexano, acetato de etila e metanol. As frações foram analisadas por cromatografia em camada fina (usando como eluentes misturas de solventes próximos aos que as frações haviam saído da coluna) e reunidas por semelhança. As frações resultantes foram então submetidas à espectroscopia de ressonância magnética nuclear de hidrogênio $\left(\mathrm{RMN}{ }^{1} \mathrm{H}\right)$ e carbono (RMN ${ }^{13} \mathrm{C}$ ) usando clorofórmio deuterado como solvente para identificar as substâncias isoladas. A identificação dos triterpenos se deu por comparação dos dados espectrométricos obtidos com aqueles publicados em literatura especializada ${ }^{19}$. O processo de isolamento das substâncias está resumido no fluxograma 1 .

\section{Ácido Betulínico:}

p.f. $=250-255^{\circ} \mathrm{C}$

$\mathrm{RMN}^{1} \mathrm{H}\left(200 \mathrm{MHz}, \mathrm{CDCl}_{3}, \mathrm{TMS}\right)$ : (ppm) 0,$66 ; 0,77 ; 0,90 \mathrm{e} 0,96$ ( $s$, $\left.5 \mathrm{X} 3 \mathrm{H} ; \mathrm{H}_{23}, \mathrm{H}_{24}, \mathrm{H}_{25}, \mathrm{H}_{26}, \mathrm{H}_{27}\right), 1,62\left(s, 3 \mathrm{H}, \mathrm{H}_{30}\right), 2,97\left(m, 1 \mathrm{H}, \mathrm{H}_{3}\right)$, $4,55\left(s l, 1 \mathrm{H}, \mathrm{H}_{29 \mathrm{a}}\right), 4,68\left(s l, 1 \mathrm{H}, \mathrm{H}_{29 b}\right), 12,0$ ( $s l, 1 \mathrm{H}, \mathrm{H}$ ácido). RMN ${ }^{13} \mathrm{C}\left(50 \mathrm{MHz}, \mathrm{CDCl}_{3}, \mathrm{TMS}\right)$ : (ppm) 14,3 $\left(\mathrm{C}_{27}\right), 15,7\left(\mathrm{C}_{24}\right)$, $15,8\left(\mathrm{C}_{25}\right), 15,9\left(\mathrm{C}_{26}\right), 18,0\left(\mathrm{C}_{6}\right), 19,0\left(\mathrm{C}_{30}\right), 20,5\left(\mathrm{C}_{11}\right), 25,1\left(\mathrm{C}_{12}\right), 27,2$ $\left(\mathrm{C}_{2}\right), 28,0\left(\mathrm{C}_{23}\right), 29,1\left(\mathrm{C}_{21}\right), 30,1\left(\mathrm{C}_{15}\right), 31,7\left(\mathrm{C}_{16}\right), 34,0\left(\mathrm{C}^{7}\right), 36,7$ $\left(\mathrm{C}_{22}\right), 37,6\left(\mathrm{C}_{10}\right), 38,3\left(\mathrm{C}_{13}\right), 38,5\left(\mathrm{C}_{1}\right), 38,6\left(\mathrm{C}_{4}\right), 40,3\left(\mathrm{C}_{8}\right), 41,9\left(\mathrm{C}_{14}\right)$, $46,6\left(C_{18}\right), 48,5\left(C_{19}\right), 49,9\left(C_{9}\right), 54,9\left(C_{5}\right), 55,4\left(C_{17}\right), 78,1\left(C_{3}\right), 109,5$ $\left(\mathrm{C}_{29}\right), 150,2\left(\mathrm{C}_{20}\right), 180,7\left(\mathrm{C}_{28}\right)$.

EM-IE m/z (\%): $470[\mathrm{M}]+.(3,3), 452(1,1), 411(5,5), 262(33,3), 233$ $(7,7), 207(35,5), 189(66,7), 175(26,6), 133(26,6), 119(41,1), 107$ $(44,4), 95(50,0), 69(65,5), 55(93,3), 43(100)$. 


\section{Ácido Ursólico:}

$\mathrm{RMN}^{13} \mathrm{C}\left(50 \mathrm{MHz}, \mathrm{CDCl}_{3}, \mathrm{TMS}\right)$ : (ppm) 15,5 ( $\left.\mathrm{C}_{24}\right), 15,7\left(\mathrm{C}_{25}\right)$, 16,9 $\left(\mathrm{C}_{26}\right), 16,9\left(\mathrm{C}_{29}\right), 18,4\left(\mathrm{C}_{6}\right), 21,2\left(\mathrm{C}_{30}\right), 23,3\left(\mathrm{C}_{11}\right), 23,6\left(\mathrm{C}_{27}\right), 24,3$ $\left(\mathrm{C}_{16}\right), 27,3\left(\mathrm{C}_{2}\right), 28,2\left(\mathrm{C}_{15}\right), 28,2\left(\mathrm{C}_{23}\right), 30,7\left(\mathrm{C}_{21}\right), 33,0\left(\mathrm{C}_{7}\right), 36,7$ $\left(\mathrm{C}_{22}\right), 37,0\left(\mathrm{C}_{10}\right), 38,8\left(\mathrm{C}_{1}\right), 38,8\left(\mathrm{C}_{4}\right), 38,8\left(\mathrm{C}_{20}\right), 39,1\left(\mathrm{C}_{19}\right), 39,6\left(\mathrm{C}_{8}\right)$, $42,0\left(\mathrm{C}_{14}\right), 47,5\left(\mathrm{C}_{9}\right), 48,1\left(\mathrm{C}_{17}\right), 52,8\left(\mathrm{C}_{18}\right), 55,4\left(\mathrm{C}_{5}\right), 78,8\left(\mathrm{C}_{3}\right), 125,5$ $\left(\mathrm{C}_{12}\right), 138,0\left(\mathrm{C}_{13}\right), 180,0\left(\mathrm{C}_{28}\right)$.

\section{Ácido Oleanólico:}

$\mathrm{RMN}^{13} \mathrm{C}\left(50 \mathrm{MHz}, \mathrm{CDCl}_{3}, \mathrm{TMS}\right):(\mathrm{ppm}) 15,3\left(\mathrm{C}_{25}\right), 15,6\left(\mathrm{C}_{24}\right)$, 16,8 $\left(\mathrm{C}_{26}\right), 18,3\left(\mathrm{C}_{6}\right), 23,1\left(\mathrm{C}_{11}\right), 23,4\left(\mathrm{C}_{16}\right), 23,6\left(\mathrm{C}_{30}\right), 26,0\left(\mathrm{C}_{27}\right), 27,4$ $\left(\mathrm{C}_{2}\right), 27,7\left(\mathrm{C}_{15}\right), 28,1\left(\mathrm{C}_{23}\right), 30,6\left(\mathrm{C}_{20}\right), 32,3\left(\mathrm{C}_{22}\right), 32,6\left(\mathrm{C}_{7}\right), 33,1$ $\left(\mathrm{C}_{29}\right), 33,8\left(\mathrm{C}_{21}\right), 37,0\left(\mathrm{C}_{10}\right), 38,5\left(\mathrm{C}_{1}\right), 38,7\left(\mathrm{C}_{4}\right), 39,3\left(\mathrm{C}_{8}\right), 41,3\left(\mathrm{C}_{18}\right)$, $41,6\left(\mathrm{C}_{14}\right), 45,8\left(\mathrm{C}_{19}\right), 46,6\left(\mathrm{C}_{17}\right), 47,6\left(\mathrm{C}_{9}\right), 55,2\left(\mathrm{C}_{5}\right), 78,7\left(\mathrm{C}_{3}\right), 122,1$ $\left(\mathrm{C}_{12}\right), 143,4\left(\mathrm{C}_{13}\right), 181,0\left(\mathrm{C}_{28}\right)$.

\section{Referências}

${ }^{1}$ Bordignon, S.A.L. O Gênero Hyptis Jacq. (Labiatae) no Rio Grande do Sul. Campinas, Dissertação de Mestrado, Instituto de Biociências, Universidade Federal do Rio Grande do Sul, 1990.

${ }^{2}$ Menezes, F.S. Raphiodon echinus e Marsypianthes chamaedrys (Lamiaceae): Aspectos químicos e farmacológicos. Rio de Janeiro, Dissertação de Doutorado, Núcleo de Pesquisas de Produtos Naturais, Universidade Federal do Rio de Janeiro, 1997.

${ }^{3}$ Pereda-Miranda, R.; Delgado, G. Chemical studies on Mexican Hyptis species. Part 2. Triterpenoids and flavonoids from Hyptis albida. J. Nat. Prod., v. 53, n. 1, p. 182-185.

${ }^{4}$ Barbosa P.P.P.; Barbosa, C.P. Studies on the antiulcerogenic activity of the essencial oil of Hyptis mutabilis Briq. In rats. Phytotherapy Res., v. 6, n. 2, p. 114-115, 1992.

${ }^{5}$ Pereda-Miranda, R.; Gascon-Figueroa, M. Chemistry of Hyptis mutabilis: New pentacyclic triterpenoids. J. Nat. Prod., v. 51, n. 5, p. 996-998, 1988.

${ }^{6}$ Pereda-Miranda, R.; Hernandez, L.; Villavicencio, M.J.; Novelo, M.; Ibarra, P.; Chai, H.; Pezzuto, J.M. Structure and stereochemistry of pectinolides A-C, novel antimicrobial and cytitoxic 5,6-dihydro- $\alpha$ - pyrones from Hyptis pectinata. J. Nat. Prod., v. 56, n. 4, p. 583-593, 1993.

${ }^{7}$ Rojas, A.; Hernandez, L.; Pereda-Miranda, R.; Mata, R. Screening for antimicrobial activity of crude drug extracts and pure natural products from Mexican medicinal plants. J. Ethnopharmacol., v. 35, n. 3, p.275-283, 1992.

${ }^{8}$ Novelo, M.; Cruz, J.G.; Hernandez, L.; Pereda-Miranda, R.; Chai, H.; Mar, W.; Pezzuto, J.M. Chemical studies on Mexican Hyptis species. VI. Biologically active natural products from Mexican medicinal plants. II. Cytotoxic constituents from Hyptis verticillata. J. Nat. Prod., v. 56, n. 10, p. 1728-1736, 1993.

${ }^{9}$ Vega-Carrillo, H.R.; Iskander, F.Y.; Manzanares-Acuna, E. Elemental distribution in medicinal plants commonly used in folklore medicine in Mexico. Int. J. Environ. Anal. Chem., v. 66, n. 2, p. 95-105, 1997.

${ }^{10}$ Misra, T.N.; Singh, R.S.; Ojha, T.N.; Upadhyay, J. Chemical constituents of Hyptis suaveolens. Part I. Spectral and biological studies on a triterpene acid. J. Nat. Prod., v. 44, n. 6 , p. 735-738, 1981.

${ }^{11}$ Mukherjee, K.S.; Mukherjee, R.K.; Ghosh, P.K. Chemistry of Hyptis suaveolens: a pentacyclic triterpene. J. Nat. Prod., v. 47, n. 2, p. 377-378, 1984.

${ }^{12}$ Lee, K.H.; Lin, Y.M.; Wu, T.S.; Zhang, De C.; Yamagishi, T.; Hayashi, T.; Hall, I.H.; Chang, J.J.; Wu, R.Y.; Yang, T.H. Antitumor agents. LXXXVIII. The cytotoxic principles of Prunella vulgaris, Psychotria serpens, and Hyptis capitata: ursolic acid and related derivatives. Planta Med., v. 54, n. 4, p. 308-311, 1988.

${ }^{13}$ Almtorp, G.T.; Hazell, A.C.; Torssell, K.B.G. A lignan and pyrone and other constituents from Hyptis capitata. Phytochemistry, v. 30, n. 8, p. 2753-2756, 1991.

${ }^{14}$ Porter, R.B.R.; Reese, P.B.; Williams, L.A.D.; Williams, D.J. Acaricidal and insecticidal activities of cadina-4, 10(15)-dien3-one. Phytochemistry, v. 40, n. 3, p. 735-738, 1995.

${ }^{15}$ Onayade, O.A.; Looman, A.; Scheffer, J.J.C.; Svendsen, A.B. Composition of the herb essential oil of Hyptis spicigera Lam. Flavour Fragrance J., v. 5, n. 2, p. 101-105, 1990.

${ }^{16}$ Malan, K.; Pelissier, Y.; Marion, C.; Blaise, A.; Bessiere, J.M. The essential oil of Hyptis pectinata. Planta Med., v. 54, n. 6, p. 531-532, 1988.

${ }^{17}$ Aycard, J.P.; Kini, F.; Kam, B.; Gaydou, E.M.; Faure, R. Isolation and identification of spicigera lactone: complete proton and carbon-13 assignments using two-dimensional NMR experiments. J. Nat. Prod., v. 56, n. 7, p. 1171-1173, 1993.

${ }^{18}$ Zoghbi, M.D.B.; Andrade, E.H.A; da Silva, M.H.L.; Maia, J.G.S.; Luz, A.I.R.; da Silva, J.D. Chemical variation in the essential oils of Hyptis crenata Pohl. ex Benth. Flavour Fragrance J., v. 17, n. 1, p. 5-8, 2002.

${ }^{19}$ Mahato, S.B.; Kundu, A.P. Triterpenes. Phytochemistry, v. 37, n. 6, p. 1517-1575, 1994.

\section{*Autor para correspondência:}

Prof. Dr. Fábio de Souza Menezes.

Departamento de Produtos Naturais e Alimentos

Faculdade de Farmácia

Centro de Ciências da Saúde

Universidade Federal do Rio de Janeiro

Bloco A - $2^{\circ}$ andar - Sala 4 - Cidade Universitária

CEP 21941-590 - Rio de Janeiro (RJ)

E-mail: fsmenezes@ pharma.ufrj.br 